\title{
Utility of polymerase chain reaction technique in the diagnosis of tubercular meningitis in Indian children
}

\section{Baveja C .P. ${ }^{1}$, Singh T. ${ }^{1}$, Talukdar B. ${ }^{1}$, Sharma V.K.${ }^{1}$, G upta R .K. ${ }^{3}$ and R akshit $\mathbf{P}^{2 *}$}

\author{
${ }^{1}$ Dept. of Microbiology, Maulana Azad Medical College, New Delhi, India. \\ ${ }^{2}$ National Centre for Disease Control, New Delhi, India \\ ${ }^{3}$ Dept. of Microbiology, Punjab University, Chandigarh, India
}

\begin{abstract}
A bstract: Purpose: Despite tremendous advancement, tuberculosis remains a major global health problem due to multidrug resistance and HIV infection. In children's, tubercular meningitis (TBM) is the main presentation than that of pulmonary as after disease neurological problems, are more serious. Timely and accurate diagnosis is important for correct treatment and better outcome. The conventional methods (microscopy and culture) are reported either to give false results or fail to respond at early stage. The molecular biology techniques have offered a ray of hope over it. Methods: In the present study, 30 suspected cases of TBM along with 15 controls representing other forms of meningitis and of pediatric age group were diagnosed using PCR and conventional methods. Results: Only two samples showed positivity by microscopy and six samples showed positivity by culture. On the other hand by PCR, a significantly higher number (17, including microscopy and culture positive) were positive for the presence of TBM. All the control samples were negative by both the methods. Conclusions: PCR was able to detect additional 11 cases reportedly negative by culture and microscopy. It shows that a big quantum left undiagnosed and hence untreated. These results suggest that PCR definitely have an advantage over conventional methods especially in case of TBM where non-treatment due to poor diagnosis may lead to high morbidity and mortality. More emphasis needs to be given to the adoption of such rapid diagnostic methods in case of infections where the start of treatment may affect the infection outcome.
\end{abstract}

K eywords: Acid fast bacilli, tubercular meningitis, cerebrospinal fluid, polymerase chain reaction

*Author for Correspondence. E-mail: partho_rakshit@yahoo.com www. ijamicro.com 


\section{Introduction}

Tremendous progress has been made in tuberculosis research since the first demonstration of the causative agent, Mycobacterium tuberculosis by Sir Robert Koch in 1882. Despite that tuberculosis remains a major global public health problem with considerable morbidity and mortality, till date [1-2]. The presentation of tuberculosis is more critical especially in developing countries, affecting both sex and adults and children's. In adults, the predominant manifestation of tuberculosis is the pulmonary form. However, in children's the extra pulmonary forms of tuberculosis are more common [2]. The most common extra pulmonary form is tubercular lymphadenitis followed by tubercular meningitis, which constitutes $7-12 \%$ of extra pulmonary tuberculosis in children. Tubercular meningitis (TBM) is more notorious than pulmonary as more than half of infected persons either die or follow neurological disorders [3-4]. Since the diagnosis of TBM is difficult to confirm, therefore, the disease usually remains underreported. Approximately 100,000 case of TBM occurs every year, globally with mortality rate as high as $19.3 \%$. In India, approximately $20 \%$ tuberculosis new cases occur per year with highest burden in world. Out of these, approximately $10-20 \%$ cases constitute childhood TB, to which approximately $25 \%$ accounts for TBM [5-6]. In India, TBM continues to be an important cause of hospitalization with considerable rate of deaths and neurological disabilities. Without treatment, virtually all patients with TBM die. However, the pathogenesis of TBM is not fully understood and hence accurate and early diagnosis becomes of much importance to start the treatment. The fortune of TBM necessitates the early and most importantly the accurate diagnosis of infection at an early stage. The diagnosis of TBM is always presumptive and mostly relies on presentation of clinical symptoms, tuberculin testing, cerebrospinal fluid findings, tomography scan, and the response to anti-tuberculosis drugs. Low sensitivity and slow growth hampers the diagnosis at an early stage $[4,7-8]$. Therefore, high degree of suspicion is justified for the diagnosis of TBM, which represents non-specific symptoms.

Till date, conventional methods such as microscopy and culture have been gold standard for the diagnosis of tuberculosis. A direct acid-fast bacilli smear from cerebrospinal fluid is of diagnostic value but usually also suffers with false negative and low positive results of suspected TBM cases. Further, culture requires long incubation period of 6-8 weeks and is often negative in result $[4,7-8]$. Number of other methods as adenosine deaminase assay, RIA, and ELISA has also been used previously for the diagnosis of TBM [3, 9-12]. However, all these methods have been shown to be associated with either low sensitivity or specificity. There has been a concurrent demand for the consistent and rapid method for the diagnosis of TBM. In this regard, molecular methods based on DNA/RNA have been proven to a good choice as they can detect as low as 2-3 organisms [3]. Amongst various methods, polymerase chain reaction (PCR) based methods are rapid, easy, cost effective, sensitive and specific as compared to other tests [3-10, 13-14]. Despite its advantages over conventional methods, which usually result in false positive/negative results, the PCR technique has not been adopted in the diagnosis of TBM, yet. Therefore, the present study was planned to evaluate usefulness of PCR in the diagnosis of clinically suspected TBM cases in the pediatric age group and its comparison with conventional techniques, in a tertiary care hospital of North India. 


\section{M aterials and M ethods}

Study Subjects and Samples: The study was conducted in the Department of Microbiology, Maulana Azad Medical College in collaboration with department of pediatrics, Lok Nayak Hospital, New Delhi. Institutional Ethical Committee approved the study protocol. Leftover samples of cerebrospinal fluid from 30 clinically suspected cases of TBM (16 males and 14 females; age range from 6 months to 11years), received in a sterile leak proof containers for routine diagnostics was used. Samples were centrifuged at $3600 \mathrm{Xg}$ for 30 minutes to concentrate. Supernatant was decanted and sediment was vortexed thoroughly and divided into three aliquots; one each for microscopy, culture and PCR analysis. A detailed history of subjects was taken including clinical signs and symptoms, family history, immunization status, past history of tuberculosis etc. according to the criteria as adopted previously for the clinical suspected TBM (Table 1) [3]. 15 subjects with similar age and sex groups representing other forms of meningitis were taken as control.

Microscopy and Culture: One part of sample was processed for microscopy according to standard procedures of Zeihl- Neelsen stain, Kinyoun stain and Auramine-O stain. Another part of specimen was cultured on Lowenstein Jensen and Middle brook 7H10 medium. The entire tests were performed according to standard procedures [15]. Positive samples were further subjected to niacin and nitrate reduction test.

DNA Extraction for PCR: DNA from cerebrospinal fluid samples was extracted according to the method described earlier [16]. Briefly, $200 \mu 1$ of sample was mixed with $200 \mu 1$ of lysis buffer (5.3M Guanidine isothionate, 10mM Diethyribiol, 1\% Tween 20, 0.3M Sodium acetate, 50mM Sodium citrate). Mixture was incubated at $65^{\circ} \mathrm{C}$ for 10 minutes followed by centrifugation at $1600 \mathrm{~g}$ for 1 minute. Supernatant was discarded and pellet was re-suspended in wash buffer $(50 \%$ ethanol, $10 \mathrm{mM}$ Tris $\mathrm{HCl}, 100 \mathrm{mM} \mathrm{NaCl}$ ). Finally, DNA was eluted with mixing of $10 \mathrm{mM}$ Tris $\mathrm{HCl}$. DNA was washed in $70 \%$ ethanol, dried and was re-suspended in $20 \mu 1$ Tris EDTA. DNA was stored at $-20^{\circ} \mathrm{C}$ till further use.

PCR Amplification for TBM : A 240 base pair region from the gene coding the MPB64 protein was selected for amplification since it is present only in members of $M$. tuberculosis complex. Moreover, Indian strains have also been reported to have poor sensitivity with IS6110 PCR. For amplification, forward primer 5"-TCCGCTGCCAGTCGTCTTCC-3" and reverse primer 5"GTCCTCGCGAGTCTAGGCCA-3" were used. Amplification was done in $25 \mu 1$ reaction mixture containing 10ng of genomic DNA, 10mM Tris HCL (pH 8.0), $1.5 \mathrm{mM} \mathrm{MgCl}_{2}, 50 \mathrm{mM} \mathrm{KCl}, 200 \mu \mathrm{M}$ of each dNTPs, 5pmole of each primer and $0.5 \mathrm{U}$ of Taq DNA polymerase (Bio Labs, New England) using DNA thermal cycler (MJ Research, USA). Cycles used were, initial denaturation at $94^{\circ} \mathrm{C}$ for 4 minutes followed by 30 cycles at $94^{\circ} \mathrm{C}$ for 30 seconds, $60^{\circ} \mathrm{C}$ for 60 seconds and $72^{\circ} \mathrm{C}$ for 30 seconds. Final extension was done at $72^{\circ} \mathrm{C}$ for 5 minutes. Amplified PCR products were electrophoresed on $2 \%$ ethidium bromide stained agarose gel along with Gene Rule 100bp DNA ladder. Positive and negative controls were also run along with the samples. Gel was visualized for amplified product and was photographed under UV transilluminator. 
Statistical Analysis: SPSS Version 16.0 was used for statistical analysis. Chi square test carried out and value less than 0.05 were considered statistically significant.

\section{Results}

In the present study, cerebrospinal fluid was collected from 30 cases of clinically suspected TBM and 15 controls of other suspected meningitis cases. Samples were processed for microscopy, culture, and PCR. Out of 30 samples, only 2 samples were positive by microscopy including one each with Zeihl-Neelsen and Kinyoun. These 2 samples were also positive by fluorescent stain Auramine-O. On culture on Lowenstein Jensen and Middlebrook 7H10 media, 6 samples showed bacterial growth including 2 sample positive by microscopy (Table 2). None of the control sample was found positive with any of the aforesaid methods.

On PCR analysis, significantly higher numbers of samples were found to be positive for the presence of TBM including the samples found positive by microscopy and culture ( $\mathrm{p} \leq 0.001$, Table 2, Figure 1). Additionally, 11 cases which were negative earlier by culture and microscopy, also showed presence of TBM by PCR analysis (Table 2). Out of 30 cases, 13 samples were negative for the presence of TBM by PCR as well as culture and microscopy. All the control samples were also negative for the presence of TBM by all the methods used.

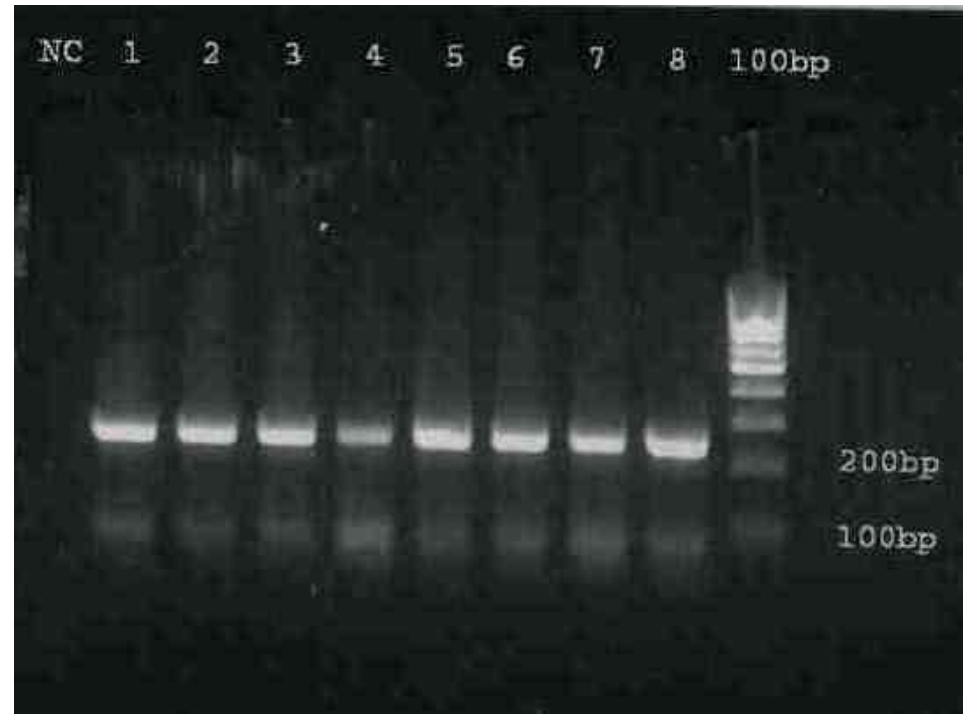

Figure 1: Representative photomicrograph showing the presence of 240bp amplified product in TBM positive samples as compared to 100bp DNA ladder. Negative control shows the absence of any DNA band. 
Table 1: Criterion adopted for clinical suspicion of tubercular meningitis for inclusion of paediatric subject in study.

\begin{tabular}{|c|l|}
\hline \multirow{4}{*}{\begin{tabular}{c} 
Criteria for \\
TBM inclusion \\
\cline { 2 - 2 }
\end{tabular}} & $\begin{array}{l}\text { History of prolonged fever, headache, vomiting, stiff neck, irritability and gradual loss } \\
\text { of plactivity, neurological deficit } \\
(>60 \%), \text { proteins }(>100 \mathrm{mg} \%) \text {, sugars }(<60 \%)\end{array}$ \\
\cline { 2 - 3 } & Close contact (at least 2 days per week) with a confirmed active tuberculosis patient \\
\cline { 2 - 3 } & Positive Mantoux test (i.e. induration $>10 \mathrm{~mm})$ \\
\cline { 2 - 3 } & Microbiological and radiographic evidence of active tuberculosis at an extraneural site \\
\cline { 2 - 3 } & $\begin{array}{l}\text { Cranial computerized tomographic scan showing central nervous system densities or } \\
\text { basal exudates. }\end{array}$ \\
\hline
\end{tabular}

Table 2: Comparison of polymerase chain reaction results with those obtained by conventional diagnostic methods.

\begin{tabular}{|c|c|c|c|}
\hline Sr. No. & Conventional Methods & PCR Positive & PCR Negative \\
\hline 1. & $\begin{array}{c}\text { Microscopy positive } \\
\text { Culture positive (n=2) }\end{array}$ & 2 & 0 \\
\hline 2. & $\begin{array}{c}\text { Microscopy negative } \\
\text { Culture positive (n=4) }\end{array}$ & 4 & 0 \\
\hline 3. & $\begin{array}{c}\text { Microscopy negative } \\
\text { Culture negative (n=24) }\end{array}$ & 11 & 13 \\
\hline & Total $\quad(n=30)$ & 17 & 13 \\
\hline
\end{tabular}

\section{Discussion and Conclusion}

TBM is the commonest most severe clinical manifestations of extra-pulmonary tuberculosis [17]. The early and proper treatment solely depends upon accurate diagnosis for improved outcome. However, the diagnosis of TBM is controversial since the clinical symptoms are non--specific [4, 17]. In an earlier study, it was found that $20 \%$ of paediatric patients, who died of active tuberculosis, had CNS involvement [18]. Till date, the diagnosis of TBM is made by acid fast bacilli staining and culture. However, many reports have indicated positive results only in $8-10 \%$ and $29-48 \%$ cases for AFB and culture, respectively [19]. In the Indian subcontinent, smear and culture positivity has been found to be in $15-20 \%$ cases only $[3,20]$. In the present study, all 30 subjects were either in 2nd or 3rd stage of TBM. As control, 15 subjects of same age and sex representing other forms of meningitis were also included. The aseptically collected cerebrospinal fluid from subjects and controls was processed for microscopy, culture, and PCR test. 
The results of microscopy showed presence of bacilli by different staining methods as ZeihlNeelsen (3.3\%), Kinyoun (3.3\%) and Auramine O (6.7\%). The use of fluorescent stain further improved the sensitivity of the microscopy by $3.4 \%$. It has been reported previously that rate of Zeihl-Neelsen positivity varies from $10 \%$ to $40 \%$. Few studies have also reported positivity as low as $5 \%$ or less. The results of present study corroborates well with earlier reported findings that have shown positivity rate of only $3 \%[1,4,12,21-23]$. It has been documented that rate of success with Zeihl-Neelsen stain varies with volume of cerebrospinal fluid sample. The larger the sample volume, greater chances to get the positive results ${ }^{[15]}$. However, in the current study only one sample was positive due to the small volume of samples collected from the paediatric age group. The fluorescent stain increased the sensitivity by $100 \%$ to acid fast stain, as reported previously $[1,23]$.

In tuberculosis, culture has been observed as the gold standard but the chances of culture positivity are less in paediatric age group due to paucibacillary nature of the disease. In the present study, $20 \%$ cases were found positive by Lowenstein-Jensen and Middle brook $7 \mathrm{H} 10$ media culture. The samples positive by microscopy were also found to be positive in culture and hence confirm the status of TBM. Nevertheless, the culture detected $13.3 \%$ additional cases, which were negative by microscopy. Various previous studies have also demonstrated the similar culture positivity [22, 24-26]. Similar to acid fast staining, the incidence of culture positivity also increases with repeat culture especially with large volume of cerebrospinal fluid samples. However, variable rate of culture positivity in have also been reported $[8,13,27]$.

The molecular biology techniques have been used in the diagnosis of tuberculosis since the use of DNA probes. [28] The sensitivity and specificity of PCR assay rely upon set of primer used. Many different set of primers have been used for the detection of Mycobacterium tuberculosis by PCR assay. Most of these primers were based on gene IS6110. The IS6110 sequence has been selected due to its multiple copies in the most strains of M. tuberculosis genome [29-30]. Assuming that multiple copies will confer higher sensitivity in PCR assay for the diagnosis of TBM, majority of the investigations have been performed based on insertion sequence IS6110 as a target [2, 31-33]. However, it is not reliable since M. tuberculosis strains lacking IS6110 have also been reported from India and hence indicates poor PCR sensitivity [29-30]. Therefore, in the present study, a part of DNA coding a specific protein MPB64 and present only in members of $M$. tuberculosis complex (M. tuberculosis, M. bovis and strains of BCG) and prevalent in India was selected for the amplification purpose. In a study reported from India, PCR for the $38 \mathrm{kDa}$ gene as the target sequence showed the detection sensitivity as low as 10fg DNA, which is equivalent to the two to three organisms only, and hence proves to be highly sensitive and specific [3]. In the present study, a total of $56.7 \%$ cases were found positive with PCR. The PCR positive cases include the microscopy and culture positive patients. Additionally, PCR was sensitive enough to detect $36.7 \%$ TBM suspected cases, which were otherwise negative by culture and microscopy. The result suggests that PCR assay was more sensitive, and specific enough to detect TBM in paediatric population as compared to conventional methods. It further indicates that in the absence of PCR assay, many suspected cases would have been left undetected and hence untreated. Previously, a number of studies have also shown the usefulness of PCR over 
conventional assays for the diagnosis of tuberculosis. In a study carried out in India, more specific and sensitive $38 \mathrm{KDa}$ gene was targeted in PCR assay for the detection of TBM. It was found to be more specific and sensitive as compared to microscopy, BactT and Lowenstein-Jensen culture [13]. In another study, real time PCR has been shown more sensitive and rapid method for the diagnosis of TBM. The conventional methods were shown to be less sensitive than PCR and it was suggested that PCR can be adopted for the routine diagnosis of TBM. [4, 7-8] In a yet another study, 162 patients cerebrospinal fluid samples was evaluated by real time PCR assay for the early and specific diagnosis of TBM [2]. Various other studies have also indicated the higher positivity rates of PCR assay over the conventional methods. In the present study, the sample size and the sample volume may account for the sensitivity of polymerase chain reaction towards the lower side. But the evidence available through multiple studies, the usefulness of PCR assay over others cannot be overlooked. The PCR assay may be explored at a large level to make it acceptable as standard parameter for the diagnosis of TBM, at least for paediatric age group, where early diagnosis and treatment reflects in patient survival.

\section{Acknowledgement}

Authors gratefully acknowledge unknown group population constituting all the subjects, belonging to different areas of New Delhi. For the study purpose, the leftover sample of routine diagnostics was used.

C onflicts of Interest: None to be declared.

\section{References}

1. Pai, M., Flores, L.L., Pai, N., 2003, "Diagnostic accuracy of nucleic acid amplification tests for tuberculous meningitis: a systematic review and meta-analysis", Lancet Infect. Dis., 3(10), 63343.

2. Shirani, K., Zahra, T., Majid, Y., 2015, "Diagnosed tuberculous meningitis using cerebrospinal fluid polymerase chain reaction in patients hospitalized with the diagnosis of meningitis in referral hospitals in Isfahan”, J. Res. Med. Sci., 20(3), 224-227.

3. Kulkarni, S., Sola, C., Filliol, I., 2005, "Spoligotyping of Mycobacterium tuberculosis isolates from patients with pulmonary tuberculosis in Mumbai, India”, Res. Microbiol., 156(4), 588-96.

4. Török, M.E., 2015, "Tuberculous meningitis: advances in diagnosis and treatment", Br. Med. Bull., 113(1), 117-31.

5. Wilkinson, R.J,, Ursula, R., Usha, K.M., Reinout, C., Nguyen, T.H.M., Kelly, E.D., Maxine, C., Anthony, F., Rada, S., Regan, S., Guy, E.T., 2017, “Tuberculous meningitis”, Nature Rev. Neurol., 13, 581-598.

6. Israni, A.V., Dave, D.A., Mandal, A.S., Sahi, P.K., Das, R.R., Shah, A, 2016, "Tubercular meningitis in children: Clinical, pathological, and radiological profile and factors associated with mortality", J. Neurosci. Rural. Pract., 7(3), 400-404. 
7. Marx, G.E., Chan, E.D., 2011, "Tuberculous Meningitis: Diagnosis and Treatment Overview," Tuberculosis Res. Treatment, 798764.

8. Neelakantan, S., Amita, J., Pooja, S, 2014, "Performance of newer and conventional diagnostic methods in detection of drug sensitive and resistant tuberculous meningitis", Asian Pac. J. Trop. Dis., 4(S2), S648-S652.

9. Marais, B.J., Pai, M., 2006, "Specimen collection methods in the diagnosis of childhood tuberculosis", Ind. J. Med. Microbio., 24(4),249-251.

10. Marais, B.J., Pai, M., 2007, "New approaches and emerging technologies in the diagnosis of childhood tuberculosis", Paed. Resp. Rev., 8(2), 124-133.

11. Kusum, S., Aman, S., Pallab, R., 2011, "Multiplex PCR for rapid diagnosis of tuberculous meningitis", J. Neurol., 258(10),1781-7.

12. Rock, R.B., Olin, M., Baker, C.A., 2008, "Central nervous system tuberculosis: pathogenesis and clinical aspects”, Clin. Microbiol. Rev., 21(2),243-61.

13. Singh, A.K., Xavier, C., Lakshmi, P., 2016, "Investigating essential gene function in Mycobacterium tuberculosis using an efficient CRISPR interference system", Nucleic Acids Res., 44 (18), e143.

14. Thwaites, G.,E., Toorn, R., Schoeman, J., 2013, “Tuberculous meningitis: more questions, still too few answers", Lancet Neurol., 12(10),999-1010.

15. Collee, J.G., Miles, R.S., Watt, B., 1996, "Tests for the identification of bacteria. In: Collee JG, Marmion BP, Fraser AG, Simmons A, editors. Mackie and Mc Cartney Practical Medical Microbiology, 14th ed., Edinburg: Churchill Livingstone, 131-50.

16. Buch, G.E., O’Hara, L.C., Summersgill, J.T., 1992, "Rapid simple method for treating clinical specimens containing $M$. tuberculosis to remove DNA for polymerase chain reaction”, J. Clin. Microbiol., 30,1331-34.

17. Principi, N., Esposito, S., 2012, "Diagnosis and therapy of tuberculous meningitis in children”, Tuberculosis, 92,377-383.

18. Udani, P.K., Dastur, D.K., 1970, "Tuberculous encephalopathy with and without meningitis. Clinical features and pathological correlations", J. Neurol. Sci., 10,541-61.

19. Stamos, J.K., Rowley, A. H., 1995, "Pediatric tuberculosis: an update", Curr. Probl. Pediatr., 25,131-136.

20. Tandon, P.N., 1978, “Tuberculous meningitis. In Handbook of Clinical Neurology”, 33, 195-262.

21. Michael, J.S., John, T.J., 2012, "Extensively drug-resistant tuberculosis in India: A review”, Ind. J. Med. Res., 136(4), 599-604.

22. Rafi, W., Venkataswamy, M.M., Nagarthana, S., 2007, "Role of an IS6110 uniplex PCR in the diagnosis of tuberculous meningitis: Experience at a tertiary neurocentre", Int. J. Tuberc. Lung Dis., 11,209-14. 
23. Jatana, S.K., Nair, M.N., Lahiri, K.K., 2000, "Polymerase chain reaction in the diagnosis of tuberculosis", Indian Pediatr., 37,375-382.

24. Smith, G.C., McDonald, R.A., Wilkinson, D., 2012, "Comparing badger (Meles meles) management strategies for reducing tuberculosis incidence in cattle", PLoS ONE, 7(6), e39250.

25. Manjunath, N., Shankar, P., Rajan, L., 1991, "Evaluation of a polymerase chain reaction for the diagnosis of tuberculosis", Tubercle., 72(1),21-7.

26. Lang, A.M., Feris-Inglesias, J., Pena, C., 1998, “Clinical evaluation of the Gen-Probe amplified direct test for the detection of Mycobacterium tuberculosis complex organisms in cerebrospinal fluid", J. Clin. Microbiol., 36,2191-4.

27. Thakur, R., Goyal, R., Sarma, S., 2010, "Laboratory diagnosis of tuberculosis meningitis - Is there a scope for further improvement?", J. Lab. Physicians, 2, 21-4

28. Grange, J.M., 1989, “The rapid diagnosis of paucibacillary tuberculosis”, Tubercle, 70(1),1-4.

29. Soolingen, V.D., Herman, P.W., de Haas, P.E., 1991, "Occurrence and stability of insertion sequence in Mycobacterium tuberculosis complex strains: Evaluation of an insertion sequencedependent DNA polymorphism as a tool in the epidemiology of tuberculosis, J. Clin. Microbiol., $29(11), 2578-89$.

30. Soolingen, V.D., de Haas, P.E., Groenen, P.M., 1993, "Comparison of various repetitive DNA elements as genetic markers for strain differentiation and epidemiology of Mycobacterium tuberculosis", J. Clin. Microbiol., 31(8), 1987-1995.

31. Miörner, H., Sjöbring, U., Nayak, P., 1995, "Diagnosis of tuberculous meningitis: a comparative analysis of 3 immunoassays, immune complex assay and the polymerase chain reaction”, Tuber. Lung Dis., 76(5), 381-6.

32. Caws, M., Wilson, S.M., 2000, "Role of IS6110 targeted PCR, culture biochemical chemical and immunologicals criteria for diagnosis of tuberculous meningitis", J. Clin. Microbiol., 38(9),31503155 .

33. Narayanan, S., Parandaman, V., Narayanan, P.R., 2001, "Evaluation of PCR using TRC4 and IS6110 primers in detection of tuberculous meningitis", J. Clin. Microbiol., 39,2006-8.

34. Narayanan, S., Das, S., Garg, P., 2002, "Molecular epidemiology of tuberculosis in a rural area of high prevalence in South India: implications for disease control and prevention", J. Clin. Microbiol., 40, 4785-4788. 